\title{
openheart Late-stage left ventricular dysfunction in adult survivors of tetralogy of Fallot repair in childhood
}

\author{
Abdel Anabtawi, Judith Mondragon, Diane Dodendorf, Warren K Laskey
}

To cite: Anabtawi A, Mondragon J, Dodendorf D, et al. Late-stage left ventricular dysfunction in adult survivors of tetralogy of Fallot repair in childhood. Open Heart 2017;4:e000690. doi:10.1136/ openhrt-2017-000690

Received 21 July 2017 Revised 24 August 2017 Accepted 7 November 2017

CrossMark

Department of Internal Medicine, University of New Mexico School of Medicine, Albuquerque, New Mexico, USA

Correspondence to DrWarren K Laskey; wlaskey@ salud.unm.edu

\begin{abstract}
Objective Left ventricular systolic dysfunction (LVSD) in adult survivors of tetralogy of Fallot (TOF) repair in childhood has been observed, although the relationship with long-term outcome remains inadequately described. Methods A cohort of 44 consecutive adult patients with TOF repair in childhood were followed prospectively from January 2001 through June 2016. LVSD was defined as an echocardiographically derived left ventricular (LV) ejection fraction $<0.55$. Clinical and demographic characteristics in patients with and without LVSD were compared. Event-free survival (all-cause death or hospitalisation) was estimated using the product limit method.

Results The average time from childhood surgical repair to cohort inception was similar between groups (LVSD, $33.7 \pm 12.7$ years; normal LV function, $36.1 \pm 14.9$ years; $\mathrm{P}=0.62$ ) as were their mean ages (LVSD, $36.5 \pm 14.5$ years; normal LV function, $40.7 \pm 15.2$ years; $P=0.73$ ). Patients with LVSD $(n=13)$ had more prior surgeries, more frequent history of significant pulmonic regurgitation, right ventricular systolic dysfunction and more implantable cardiac devices. Over a total observation time of 15.5 years, patients with LVSD were at significantly higher risk of all-cause death or hospitalisation $(\mathrm{P}=0.006)$. Onset of LVSD frequently preceded an adverse outcome.

Conclusions In this cohort of adult patients with TOF repair in childhood followed for a total of 550 patient-years, the frequency of LVSD was $30 \%$. LVSD was associated with lower event-free survival. The appearance of LVSD many years after TOF repair may herald the onset of an adverse outcome.
\end{abstract}

\section{INTRODUCTION}

The prevalence in the adult population of individuals with congenital heart disease (CHD) defects repaired in childhood has increased dramatically over the past several decades and is, appropriately, receiving increased attention. ${ }^{2}$ Tetralogy of Fallot (TOF) remains the most common cyanotic congenital heart defect occurring in about 1 in 3500 live births and accounts for between $7 \%$ and $10 \%$ of all congenital heart defects. Surgical correction in early childhood had led to high rates of event-free survival, although many individuals remain at increased risk of death or hospitalisation owing to late sequelae of these childhood procedures. ${ }^{2}$ Left ventricular systolic

\section{KEY QUESTIONS}

What is already known about this subject? Left ventricular dysfunction (LVD) has been observed in $25 \%-33 \%$ of late survivors of tetralogy of Fallot (TOF) repair in childhood and has been associated with right ventricular dysfunction and/or pulmonic regurgitation.

What does this study add?

In this long-term outcomes study, we observed the late development of LVD in 30\% of subjects with TOF followed over 16 years. The development of LVD frequently preceded and was significantly associated with the risk for death or hospitalisation.

How might this impact on clinical practice? Close follow-up of long-term survivors of TOF repair in childhood is warranted. The onset of LVD should be viewed as a potential harbinger of subsequent adverse clinical events, particularly in the presence of right ventricular dysfunction

dysfunction (LVSD) in adult survivors of TOF repair in childhood has been recognised,,$^{34}$ although its prevalence, relationship to right ventricular (RV) function and impact on late event-free survival remain areas for ongoing study. The purpose of the present study was to assess the latter issues in a prospective open cohort study design of adults with TOF repair.

\section{METHODS}

Patients with a principal diagnosis of TOF were identified from review of our registry of adults (>age 18years) with CHD referred to the University of New Mexico Adult CHD programme, currently the only certified programme in the state of New Mexico for the care of adults with CHD. A prospectively defined open cohort of consecutive patients was created in January 2001 and, for purposes of this analysis, followed through June 2016. Patients with pulmonary atresia/ ventricular septal defect, concomitant conotruncal defects, heterotaxy syndrome 
or other syndromic CHDs were excluded. Over the same time interval, we identified age-frequency-matched and sex-frequency-matched patients with atrial septal defect or isolated pulmonic valve stenosis to serve as a comparator group.

No patient was lost to follow-up over this 15.5-year interval. Ascertainment of clinical and vital status was $100 \%$. The date and nature of event rates were confirmed by review of the clinical record. Deaths were confirmed using the clinical record and the National Death Index.

LVSD was defined as an echocardiographically determined ejection fraction (EF) $<55 \%$ using the Simpson biplane method. Categories for LVSD were defined as follows: LVEF $45 \%-54 \%$, 35\%-44\% and $<35 \%$. RV size and function were qualitatively assessed from the same transthoracic echo data in accordance with the American Society of Echocardiography recommendations. ${ }^{5}$

The degree of pulmonic regurgitation was assessed also in accordance with the American Society of Echocardiography guidelines. ${ }^{6}$

Continuous data were summarised as mean \pm SD. Frequency data were summarised as counts or percentages. Comparisons between groups of continuous variables were accomplished with unpaired t-tests, while comparisons of nominal or categorical variables were accomplished with $\mathrm{X}^{2}$ statistics. The product limit approach was used for time to event analysis and results were stratified by presence of LVSD. The outcome of interest was the composite of all-cause mortality or documented hospitalisation for heart failure. Results stratified on left ventricular (LV) function group are presented graphically as Kaplan-Meier (K-M) plots and compared using log-rank statistics. All analyses were conducted using STATA/IC V.10.0. A P value $<0.05$ was considered statistically significant.

\section{RESULTS}

Four hundred and fifty unique adult patients with CHD were seen from 1 January 2001 through 30 June 2016. Forty-four patients (mean age 38.6 \pm 15 years; 19 male, 25 female) with a principal diagnosis of TOF $(9.7 \%$ of all patients with CHD) were identified and included for analysis. Fifty-two age-matched and sex-matched patients with atrial septal defect or isolated pulmonic valve stenosis served as a control group.

Table 1 summarises the basic demographic and clinical differences between patients with TOF with $(n=13$, $29.5 \%$ ) and without LVSD ( $\mathrm{n}=31,70.5 \%)$.

Of the LVSD group, 5 (38\%) were women. Forty of the $44(91 \%)$ patients with TOF had a transannular patch repair and were equally distributed between the two LV function groups. The frequency of LVSD in the control group was significantly lower than that in patients with TOF $(7.7 \%$ vs $29.5 \%$, respectively, $\mathrm{P}=0.005)$.

The mean LVEF in the LVSD group was $40 \% \pm 4 \%$, with severe or moderate LVSD in eight patients. Patients with LVSD were more likely to have right ventricular systolic dysfunction (RVSD) (Fisher's exact $\mathrm{P}=0.015$ ), more cardiac surgeries, and a history of arrhythmia and pacemaker or implantable cardiac device (ICD) placement. The indication for ICD placement was predominantly for primary prevention.

Six patients in the LSVD group $(46 \%)$ had a history of moderate to severe pulmonary regurgitation, and four patients $(31 \%)$ had undergone previous pulmonary valve replacement. A history of pulmonary regurgitation was more frequent in those with LVSD.

Importantly, no patient had LVSD at cohort inception in January 2001. The incidence density rate for LVSD was 2.36 per 100 patient-years. The estimated Kaplan-Meir survival rates over the total at-risk time of 550 patient-years

\begin{tabular}{|c|c|c|c|}
\hline Characteristics & No LVSD ( $n=31)$ & LVSD (n=13) & $P$ value \\
\hline Mean age $\pm S D$ (range), years & $40.7 \pm 15.2(24-68)$ & $36.5 \pm 14.5(20-68)$ & 0.73 \\
\hline Female, $\mathrm{n}(\%)$ & $20(65 \%)$ & $5(38 \%)$ & 0.11 \\
\hline Prior surgeries (n) & 52 (1.7 surgery/patient) & 33 (2.6 surgery/patient) & 0.03 \\
\hline With history of significant PR, n (\%) & $5(16 \%)$ & $6(46 \%)$ & 0.04 \\
\hline With history of PV replacement, $\mathrm{n}(\%)$ & $8(26 \%)$ & $4(31 \%)$ & 0.82 \\
\hline With RVSD, n (\%) & $8(26 \%)$ & $9(69 \%)$ & 0.01 \\
\hline With ICD/pacemaker, n (\%) & $2(6 \%)$ & $7(53 \%)$ & 0.01 \\
\hline $\begin{array}{l}\text { Average time } \pm S D \text { from initial repair to present analysis } \\
\text { (range), years }\end{array}$ & $36.1 \pm 14.9(2-68)$ & $33.7 \pm 12.7(19-59)$ & 0.62 \\
\hline Type II diabetes (\%) & $(6 \%)$ & $(15 \%)$ & 0.41 \\
\hline Stroke (\%) & $(0 \%)$ & $(8 \%)$ & - \\
\hline Hospital admission for heart failure, $\mathrm{n}(\%)$ & $3(10 \%)$ & $5(38 \%)$ & 0.02 \\
\hline Mean $( \pm$ SD) QRS duration, ms & $144(21.5)$ & 154 (33.2) & 0.33 \\
\hline
\end{tabular}

ICD, implantable cardiac device; LVSD, left ventricular systolic dysfunction; PR, pulmonic regurgitation; PV, pulmonic valve; RVSD, right ventricular systolic dysfunction. 


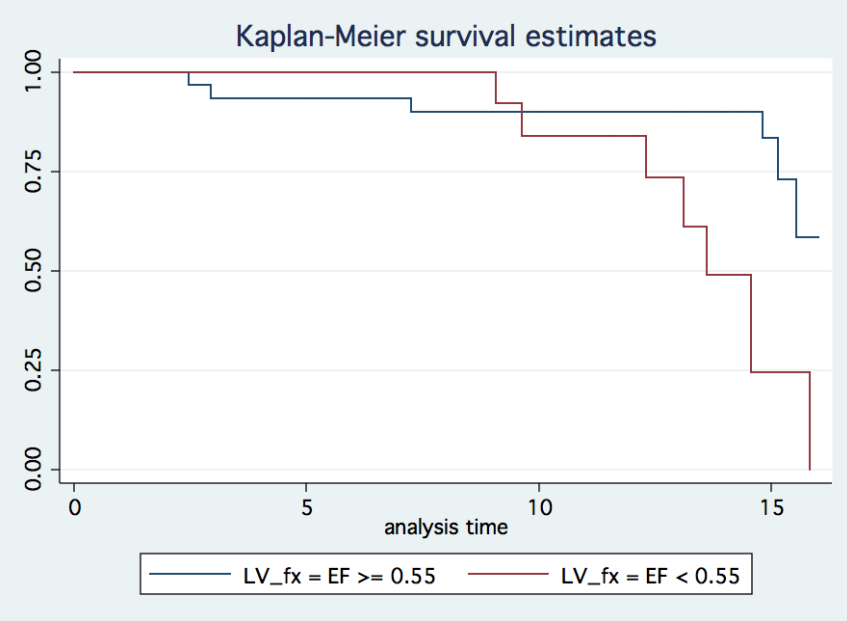

Figure 1 Time to first event analysis revealed a significant difference in estimated event-free (death or hospitalisation) survival between patients with left ventricular systolic dysfunction $\left(L V \_f x=E F<0.55\right)$ and those with preserved LV systolic function (LV_fX $=E F \geq 0.55$ ) (log-rank $\mathrm{P}=0.006)$. $E F$, ejection fraction; $L F$, left ventricular.

were significantly different for the two groups (log-rank $\mathrm{P}=0.006$; figure 1 ), with late clustering of outcomes observed in both groups. There were two deaths and five hospitalisations in the LVSD group overall, and three deaths and three hospitalisations in the preserved LV function group overall (incidence density rates: LVSD, 4.3 per 100 patient-years; preserved LV function, 1.5 per 100 patient-years). The estimated mean event-free survival was 13.5 years in the LVSD group and 14.6years in the preserved LV function group (an underestimate since the largest observed analysis time was censored).

The mean time from cohort inception to recognition of LVSD was 11.1 \pm 2.3 years (median 11.4 years) and preceded an adverse outcome by, on average, 2.6 years in five of the seven patients with an adverse outcome. In one subject LVSD was recognised at the time of the patient's demise.

\section{DISCUSSION}

In the present prospective cohort study, we observed LVSD in $30 \%$ of adult patients late after childhood TOF repair. In the majority of these patients with LVSD, LV function was moderately to severely reduced. LVSD was associated with RVSD, multiple prior surgeries, a history of significant pulmonary regurgitation, and arrhythmia and pacemaker or ICD placement. The incidence rate for LVSD was 2.36 per 100 patient-years. Of particular note is the late onset of LVSD in these patients and occurred, on average, 35-40 years following the initial repair. The primary outcome for this analysis, all-cause death or hospitalisation, was greater in LVSD, while the median event-free survival was significantly lower. Within several years of detection of LVSD, the majority of these patients succumbed to an adverse outcome.
Our results are consistent with previous singlecentre $^{7}$ and multicentre ${ }^{4}$ studies. The slightly higher prevalence of LVSD in the current report may reflect a greater extent of follow-up in a small state with only one dedicated ACHD programme. Our data are the only prospectively collected data with a prolonged cohort observation time of 15.5 years (550 patient-years). The appearance of LVSD in this cohort was a relatively late event and may serve as a harbinger of an adverse outcome over the ensuing several years.

The relationship between RV and LV systolic (dys) function is of clinical and prognostic relevance as both RVSD and LVSD are strong determinants of clinical outcomes. $^{4-9}$ Several hypotheses have been proposed, with most focusing on the deleterious effects of RV volume loading (from progressive pulmonic regurgitation) on LV function mediated by abnormal RV-LV electrical or mechanical coupling. ${ }^{710-12}$

Our study is limited by a number of factors and circumstances among which is the small sample size. New Mexico is an underpopulated, sizeable rural state with many geographical and economic constraints on access to medical care for complex conditions such as CHD. Over the last several decades, cardiac surgical services were available in New Mexico at only two institutions, and many patients were referred out of state for their initial surgery. Adherence to follow-up beyond the immediate postsurgical period, while adequate before patients reached adult years, suffered due to loss of insurability in the majority of patients, leading to substantial loss to follow-up and non-systematic re-entry into the healthcare system as adults with a wide variety of medical and non-medical issues.

However, once garnered within the ACHD programme at the university, the duration ( 15.5 years) and completeness of follow-up (100\%) represent strong points. We recognise, however, that many patients who underwent surgery early in life are unaccounted for in a study of this type owing to outmigration, death or inability to access the healthcare system after surgery. The bias inherent in such a study is inestimable as we cannot determine whether the patients in our programme are 'healthy' or 'sick' survivors without more knowledge of their clinical course after surgery and before re-entry into the healthcare system.

We did not systematically acquire more sophisticated measures of systolic or diastolic RV and LV function, for example, cardiac magnetic resonance, mainly for insurance-related reasons. However, prior investigators have noted abnormal LV strain in the presence of RV enlargement and/or dysfunction. ${ }^{813}$ The significant association between RV and LV systolic dysfunction observed in this and prior studies points to the need for further investigation of this hypothesis. Our EF cut-off for defining LVSD is derived from older adult populations, and therefore may not be applicable to younger adults with CHD. Given the relatively late onset of adverse outcomes, longer observation times might have revealed still higher rates of 
significant pulmonary regurgitation and possibly LVSD, although the current cohort represents patients surviving for greater than 50 years from reparative surgery. It is also unknown if earlier detection and correction of pulmonary regurgitation, when present, would impact the incidence of LVSD along with improvement in RV function. ${ }^{14}$

Funding Robert S Flinn Foundation at University of New Mexico.

Competing interests None declared.

Ethics approval The study, excluding public data sharing, was approved by the University of New Mexico Institutional Review Board and Human Research Protections Office.

Provenance and peer review Not commissioned; internally peer reviewed.

Open Access This is an Open Access article distributed in accordance with the Creative Commons Attribution Non Commercial (CC BY-NC 4.0) license, which permits others to distribute, remix, adapt, build upon this work non-commercially, and license their derivative works on different terms, provided the original work is properly cited and the use is non-commercial. See: http://creativecommons.org/ licenses/by-nc/4.0/

(c) Article author(s) (or their employer(s) unless otherwise stated in the text of the article) 2017. All rights reserved. No commercial use is permitted unless otherwise expressly granted.

\section{REFERENCES}

1. Warnes CA, Williams RG, Bashore TM, et al. ACC/AHA 2008 Guidelines for the Management of Adults with Congenital Heart Disease: a report of the American College of Cardiology/American Heart Association Task Force on Practice Guidelines (writing committee to develop guidelines on the management of adults with congenital heart disease). Circulation 2008;118:e714-e833.

2. Bhatt $A B$, Foster $\mathrm{E}$, Kuehl $\mathrm{K}$, et al. Congenital heart disease in the older adult: a scientific statement from the American Heart Association. Circulation 2015;131:1884-931.
3. Ghai A, Silversides C, Harris L, et al. Left ventricular dysfunction is a risk factor for sudden cardiac death in adults late after repair of tetralogy of Fallot. J Am Coll Cardiol 2002;40:1675-80.

4. Broberg CS, Aboulhosn J, Mongeon FP, et al. Prevalence of left ventricular systolic dysfunction in adults with repaired tetralogy of fallot. Am J Cardiol 2011;107:1215-20.

5. Rudski LG, Lai WW, Afilalo J, et al. Guidelines for the Echocardiographic Assessment of the Right Heart in Adults: A Report from the American Society of Echocardiography. J Am Soc Echocardiogr 2010;23:685-713.

6. Zoghbi WA, Adams D, Bonow RO, et al. Recommendations for Noninvasive Evaluation of Native Valvular Regurgitation: A Report from the American Society of Echocardiography Developed in Collaboration with the Society for Cardiovascular Magnetic Resonance. J Am Soc Echocardiogr 2017;30:305-63.

7. Knauth AL, Gauvreau K, Powell AJ, et al. Ventricular size and function assessed by cardiac MRI predict major adverse clinical outcomes late after tetralogy of Fallot repair. Heart 2008;94:211-6.

8. Diller GP, Kempny A, Liodakis E, et al. Left ventricular longitudinal function predicts life-threatening ventricular arrhythmia and death in adults with repaired tetralogy of fallot. Circulation 2012;125:2440-6.

9. Murphy JG, Gersh BJ, Mair DD, et al. Long-term outcome in patients undergoing surgical repair of tetralogy of Fallot. N Engl $J$ Med 1993;329:593-9.

10. Kempny A, Diller GP, Orwat S, et al. Right ventricular-left ventricular interaction in adults with Tetralogy of Fallot: a combined cardiac magnetic resonance and echocardiographic speckle tracking study. Int J Cardiol 2012;154:259-64.

11. Davlouros PA, Kilner PJ, Hornung TS, et al. Right ventricular function in adults with repaired tetralogy of Fallot assessed with cardiovascular magnetic resonance imaging: detrimental role of right ventricular outflow aneurysms or akinesia and adverse right-to-left ventricular interaction. J Am Coll Cardiol 2002;40:2044-52.

12. Santamore WP, Dell'Italia LJ. Ventricular interdependence: significant left ventricular contributions to right ventricular systolic function. Prog Cardiovasc Dis 1998;40:289-308.

13. van der Hulst AE, Delgado V, Holman ER, et al. Relation of left ventricular twist and global strain with right ventricular dysfunction in patients after operative "correction" of tetralogy of fallot. Am J Cardiol 2010;106:723-9.

14. van Straten A, Vliegen HW, Hazekamp MG, et al. Right ventricular function after pulmonary valve replacement in patients with tetralogy of Fallot. Radiology 2004;233:824-9. 